\title{
ZARISKI DECOMPOSITION OF B-DIVISORS
}

\author{
ALEX KÜRONYA AND CATRIONA MACLEAN
}

\begin{abstract}
Based on a recent work of Thomas Bauer's 1 reproving the existence of Zariski decompositions for surfaces, we construct a b-divisorial analogue of Zariski decomposition in all dimensions.
\end{abstract}

\section{INTRODUCTION}

The purpose of this paper is to present a generalization of Zariski decomposition on surfaces to the context of b-divisors. In particular, we provide such a decomposition for an arbitrary effective $\mathbb{Q}$-b-divisor on a normal $\mathbb{Q}$-factorial projective variety in the sense of b-divisors.

Originating in the seminal work of Zariski 22] on the structure of linear systems of surfaces, the Zariski decomposition $D=P_{D}+N_{D}$ of an effective $\mathbb{Q}$-divisor $D$ on a smooth projective surface $X$ over an algebraically closed field consists of a nef divisor $P_{D}$ and a negative cycle $N_{D}$ satisfying an orthogonality condition with respect to the intersection form on $X$. More specifically, given any effective $\mathbb{Q}$-divisor $D$, Zariski proves that there is a unique decomposition of $D$

$$
D=P_{D}+N_{D}
$$

such that $P_{D}$ is nef and $N_{D}$ is effective; $P \cdot C=0$, for any curve $C$ appearing in $\operatorname{Supp}(N)$; and if $\operatorname{Supp}(N)=C_{1} \cup \cdots \cup C_{n}$ then the intersection matrix $I\left(C_{1}, \ldots C_{n}\right)$ is negative definite. Zariski decomposition has the following useful properties.

(1) For any integer $k$, one $H^{0}(X,\lfloor k P\rfloor)=H^{0}(X,\lfloor k D\rfloor)$ (ie. $P_{D}$ "carries all sections of $D^{\prime}$ ).

(2) If the effective nef divisor $P^{\prime}$ satisfies $P^{\prime} \leq D$ then $P^{\prime} \leq P_{D}$,

Thus providing a strong tool to understand linear series on surfaces. It has been playing a distinguished role in the theory ever since, among others it is very useful for studying section rings

$$
R(X, D) \stackrel{\text { def }}{=} \oplus_{m \geq 0} H^{0}(X,\lfloor m D\rfloor) .
$$

Since $R(D)=R\left(P_{D}\right)$, Zariski decomposition allows us to reduce questions concerning $R(D)$ most notably whether it is finitely generated - to the case where $D$ is nef. As an illustration Zariski's paper contains an appendix by Mumford which uses several of Zariski's results in [22] to prove that the canonical ring of a surface of general type is finitely generated.

There is no immediate way to extend this definition to higher-dimensional varieties. Apart from an earlier attempt by Benveniste $([2$, , 3]) all proposed higher-dimensional generalizations have been based on those properties of the Zariski decomposition which make it useful for studying section rings, namely a) $P_{D}$ is nef and b) $H^{0}\left(X,\left\lfloor k P_{D}\right\rfloor\right)=H^{0}(X,\lfloor k D\rfloor)$ for all $k$.

Given an effective $\mathbb{Q}$-divisor $D$ on a variety $X$ it is easy to see that no $P_{D} \leq D$ can satisfy both properties if $D$ is non-nef but some multiple $k D$ of $D$ has no divisorial fixed locus. To get around this problem, we allow blow-ups. In 11, (see also [14) Kawamata defines a Zariski-type decomposition as follows.

Definition A. Let $D$ be a big divisor on a normal variety $X$. A rational (resp. real) Zariski decomposition of $D$ in the sense of Cutkosky-Kawamata-Moriwaki is a proper birational map $\mu: \widetilde{X} \rightarrow X$, and an effective $\mathbb{Q}$ (resp. $\mathbb{R}$ ) divisor $N_{D} \leq \mu^{*} D$ such that

During this project the first author was partially supported by the DFG-Leibniz program, the SFB/TR 45 "Periods, moduli spaces and arithmetic of algebraic varieties", and the OTKA Grant 61116 by the Hungarian Academy of Sciences. The second author obtained partial support from the BUDALGGEO Marie Curie Host Fellowship for the Transfer of Knowledge and the ANR project 3AGC.. 
(1) $P_{D}=\mu^{*} D-N_{D}$ is nef,

(2) $H^{0}\left(X,\left\lfloor\mu^{*}(k D)\right\rfloor\right)=H^{0}\left(X,\left\lfloor k P_{D}\right\rfloor\right)$ for all $k \geq 1$.

The requirement that the divisor $D$ be big forces the Zariski decomposition, if it exists, to be unique up to birational modification. Indeed, we then have

$$
N_{D}=\lim _{n \rightarrow \infty} \frac{\operatorname{Fix}(m D)}{m},
$$

by a result of Wilson's (see [15, Theorem 2.3.9] or 21]). The inclusion of real Zariski decompositions, hitherto believed to be uninteresting since real divisors very rarely have finitely generated section rings, is motivated by a counterexample of Cutkosky's [7] showing that certain divisors only have real Zariski decompositions.

As the main result of [11, Kawamata proves that if $(X, \Delta)$ is a normal klt pair such that $K_{X}+\Delta$ is big and possesses a real Zariski decomposition then its log canonical ring is finitely generated.

A subsequent counterexample of Nakayama's [16] showed that in general even real Zariski decompositions do not exist on higher-dimensional varieties.

The conditions a) and b) do not define a decomposition $D=P_{D}+N_{D}$ uniquely if $D$ is not big, even on surfaces. For example, let $E$ be an elliptic curve and set $X=\operatorname{Proj}\left(\mathcal{O}_{E} \oplus L\right)$, where $L$ is any degree-zero non-torsion line bundle on $E$. If we take $D=\operatorname{Proj}(L) \subset X$ then $D$ is nef but $H^{0}(k D)=\mathbb{C}$ for any $k$, so for any rational $0 \leq \lambda \leq 1$ the decomposition $P_{D}=\lambda D$ and $N_{D}=(1-\lambda) D$ satisfies conditions a) and b). Fujita gets around this problem by using the maximality of $P_{D}$ amongst nef sub-divisors of $D$. In $[9$ he gives the following definition.

Definition B. Let $D$ be an effective $\mathbb{Q}$-divisor on a normal variety $X$. A rational (resp. real) Fujita Zariski decomposition of $D$ is a proper birational map $\mu: \widetilde{X} \rightarrow X$, and an effective $\mathbb{Q}$ (resp. $\mathbb{R}$ ) divisor $N_{D} \leq \mu^{*} D$ such that

(1) $P_{D}=\mu^{*} D-N_{D}$ is nef,

(2) For any proper birational map $\nu: X^{\prime \prime} \rightarrow X^{\prime}$ and nef effective divisor $P^{\prime \prime} \leq \nu^{*} \mu^{*}(D)$ we have that $P^{\prime \prime} \leq \nu^{*}\left(P_{D}\right)$.

A Zariski decomposition in the sense of Fujita is automatically a Zariski decomposition in the sense of Cutkosky-Kawamata-Moriwaki.

The advent of multiplier ideals brought a certain analytic version of this concept. Precisely, Tsuji defines in [19]

Definition C. Let $L$ be a line bundle on $X$, a variety. An analytic Zariski decomposition of $L$ is a singular metric $h$ on $L$, semipositive in the sense of currents, such that for all $k, H^{0}\left(X, L^{k}\right)=$ $H^{0}\left(X, L^{k} \otimes \mathcal{J}\left(h^{\otimes k}\right)\right)$.

The motivation for this definition is as follows. Suppose that there were a Zariski decomposition $\mu^{*}(D)=P_{D}+N_{D}$ for $D$ on some birational modification $\mu: X^{\prime} \rightarrow X$. If the line bundle $\mathcal{O}\left(P_{D}\right)$ were not only nef, but actually semi-positive (a slightly stronger condition, which implies nef and is implied by ample), then we can put a semi-positive smooth metric on $\mathcal{O}\left(P_{D}\right)$. This descends to a singular semi-positive metric on $L=\mathcal{O}(D)$, and by definition of the analytic multiplier ideal, a section $\sigma \in H^{0}(X, m D)$ is contained in $H^{0}\left(X, m D \otimes \mathcal{J}\left(h^{\otimes m}\right)\right)$ if and only if $\mu^{*}(\sigma)$ is contained in $H^{0}\left(X, m P_{D}\right)$. Note that the analytic Zariski decomposition is not unique. More importantly, it is considerably weaker than its algebraic counterpart. The fact that $K_{X}$ has an analytic decomposition does not imply that the canonical ring is finitely generated.

In 8 , Demailly, Peternell and Schneider prove the following theorem: given a pseudo effective line bundle $L$ on a complex variety $X, L$ admits an analytic Zariski decomposition. Up to equivalence of singularities, the set of analytic Zariski decompositions admits a unique minimally singular member. Our work here can be seen as an algebraic version of this result. In 4, Boucksom, Favre and Johnsson consider a construction called the positive intersection product of a set of $b$-divisors: in the case where the set contains only one element, this is a Zariski-type decomposition. Their definition is the same as ours for big divisors. 
Shokurov's paper 18 and the survey article by Prokurhov accompanying it 17 contain many interesting Zariski-type decompositions, some of which work for $b$-divisors. In particular, the decomposition $\mathbf{D}=\mathbf{D}^{m}+\mathbf{D}^{e}$ defined in example 4.30 of Shokurov's paper gives us a Zariski-type decomposition for $b$-divisors. Although the definition is different, we show below that the our definition gives the same result as Shokurov's in the case where the divisor is big. However, they differ for non-big $b$-divisors.

The recent paper [4] by Boucksom, Favre and Jonsson also includes a Zariski-type decomposition of $b$-divsors. As discussed in section 3.4 of their paper, the case $n=1$ of their positive intersection products give a Zariski-type decomposition of $b$-divisors. Their definition is the same as ours in the case of big $b$-divisors.

In his original proof, Zariski concentrated on constructing the negative part $N_{D}$ using cunning linear algebra, which made for a reasonably complicated proof. In a recent work Bauer [1] gave a conceptual and very elegant construction of Zariski decompositions on surfaces using the characterization of the nef part $P_{D}$ as the maximal nef subdivisor of $D$.

It is this latter approach that we use to extend the notion of Zariski decomposition to b-divisors. We retain most of the characteristics of the higher-dimensional case with one notable exception: the positive part of a b-divisor is only a limit of b-nef b-divisors in a suitable sense. Our main result, proven as Theorem 3.1, is as follows.

Theorem D. Let $X$ be a $\mathbb{Q}$-factorial normal projective variety over an algebraically closed field of characteristic 0, D an effective $\mathbb{Q}$-b-divisor on $X$. Then there exists a unique decomposition

$$
\mathbf{D}=\mathbf{P}_{\mathbf{D}}+\mathbf{N}_{\mathbf{D}}
$$

where $\mathbf{P}_{\mathbf{D}}, \mathbf{N}_{\mathbf{D}}$ are effective b- $\mathbb{R}$ divisors on $X$, such that

(1) $H^{0}\left(X,\left\lfloor k \mathbf{P}_{\mathbf{D}}\right\rfloor\right)=H^{0}(X,\lfloor k \mathbf{D}\rfloor)$

(2) $\mathbf{P}_{\mathbf{D}}$ is a limit of b-nef b-divisors on every proper birational model $Y \rightarrow X$.

(3) For any nef b-divisor $\mathbf{P}^{\prime} \leq \mathbf{D}$ we have that $\mathbf{P}^{\prime} \leq \mathbf{P}_{\mathbf{D}}$.

A few words about the organization of this paper. First we fix notation, and review our strategy in Section 2. In Section 3 we construct the b-divisorial Zariski decomposition, and prove its properties modulo results proved later in the article. Section 4 is devoted to the construction and properties of separating blow-ups, the main technical tool of the paper.

Acknowledgments. Helpful discussion with Eckart Viehweg were appreciated, as were comments by Sebastien Boucksom.

\section{Strategy AND OVERVieW.}

All varieties are normal projective varieties, unless otherwise mentioned. An integral b-divisor D on $X$ is an element of the group

$$
\operatorname{Div}(X) \stackrel{\text { def }}{=} \lim _{\leftarrow} \operatorname{WDiv}(Y)
$$

with the limit taken over all proper birational models $f: Y \rightarrow X$ along with the induced homomorphisms $f_{*}: \operatorname{WDiv}(Y) \rightarrow \operatorname{WDiv}(X)$. To put it differently, $\mathbf{D}$ is a collection of divisors $D_{Y} \in \mathrm{WDiv}(Y)$ compatible with push-forwards. For every $Y \rightarrow X, D_{Y}$ is called the trace of $\mathbf{D}$ on $Y$, and is denoted $\mathbf{D}_{Y}$. One obtains rational and real b-divisors by tensoring with $\mathbb{Q}$ and $\mathbb{R}$, respectively.

As usual, the b-divisor of a nonzero rational function $\phi \in k(X)$ is defined as

$$
\operatorname{div}_{X}(\phi) \stackrel{\text { def }}{=} \sum_{\nu} \nu_{E}(\phi) E
$$

where $E$ runs through all geometric valuations with center on $X$. Two b-divisors are considered linearly equivalent if they differ by the b-divisor of a nonzero rational function.

One defines the associated b-divisorial sheaf $\mathcal{O}_{X}(\mathbf{D})$ by

$$
\Gamma\left(U, \mathcal{O}_{X}(\mathbf{D})\right) \stackrel{\text { def }}{=}\left\{\phi \in k(X)\left|\left(\operatorname{div}_{X} \phi+\mathbf{D}\right)\right| U \geq 0\right\} .
$$


Note that the sheaf $\mathcal{O}_{X}(\mathbf{D})$ is not coherent, however its space of global sections is finite-dimensional due to the inclusion $\mathcal{O}_{X}(\mathbf{D}) \hookrightarrow \mathcal{O}_{X}\left(\mathbf{D}_{X}\right)$. The Cartier closure of an $\mathbb{R}$-Cartier divisor $D$ on $X$ is the b-divisor $\bar{D}$ with trace $(\bar{D})_{Y} \stackrel{\text { def }}{=} f^{*} D$ on every model $f: Y \rightarrow X$. For more on the language of b-divisors the reader might wish to consult the appropriate chapter of $[5]$.

In constructing Zariski decompositions for b-divisors, we will follow the approach of Bauer [1]. To this end, we start by reviewing his proof for the surface case. Given an effective divisor $D$ on a surface $X$, Bauer sets:

$$
P_{D} \stackrel{\text { def }}{=} \max \left\{P^{\prime} \leq D, P \text { nef }\right\} .
$$

By this maximum, we mean that the coefficient of a prime divisor $E$ in $P_{D}$ is the maximum of $c_{E}\left(P^{\prime}\right)$ of the coefficients of $E$ in nef subdivisors of $D$.

Assume for the moment that $P_{D}$ is itself nef. Set $N_{D}=D-P_{D}$, which is effective by construction. If $C \in \operatorname{Supp}(N)$ and $P_{D} \cdot C>0$ then for any small positive $\epsilon, P_{D}+\epsilon C$ is still a nef subdivisor of $D$, contradicting the maximality of $P_{D}$. If $I\left(C_{1} \ldots C_{n}\right)$ is not negative definite then we can find an effective divisor $C^{\prime}$ supported on $\operatorname{Supp}(N)$ such that $C^{\prime} \cdot C_{i} \geq 0$ for all $i$. For small positive $\epsilon$, $P_{D}+\epsilon C^{\prime}$ is then a nef subdivisor of $D$, contradicting the maximality of $P_{D}$.

The important point is therefore the nefness of $P_{D}$, which follows from the following lemma.

Lemma 2.1. Let $X$ be a surface and let $D_{1} D_{2}$ be two nef effective divisors on $X$. Then $\max \left(D_{1}, D_{2}\right)$ is nef.

Proof. Let $C$ be an irreducible curve on $X$. We write $D_{1}=a_{1} C+D_{1}^{\prime}$ and $D_{2}=a_{2} C+D_{2}^{\prime}$. We may assume that $a_{1} \geq a_{2}$ so that $\max \left(D_{1}, D_{2}\right)=D_{1}+M$, where the coefficient of $C$ in $M_{1}$ is 0 . Hence

$$
\max \left(D_{1}, D_{2}\right) \cdot C=D_{1} \cdot C+M \cdot C \geq D_{1} \cdot C \geq 0 .
$$

This completes the proof of the lemma.

Obviously, this fails in higher dimensions, depending as it does on the fact that there is at most one prime divisor on a surface intersecting a given irreducible curve negatively. Our aim will be to show that on a suitable birational modification however, the statement of Lemma 2.1] remains true for $\mathbb{Q}$-divisors. This will enable us to construct Zariski decompositions for b-divisors in arbitrary dimensions.

Theorem 2.2. Let $X$ be a normal $\mathbb{Q}$-factorial variety, $D$ be an effective divisor on $X, D_{1}$ and $D_{2}$ nef effective $\mathbb{Q}$-subdivisors of $D$. There exists a birational morphism $F: Y \rightarrow X$ such that

$$
\max \left(F^{*}\left(D_{1}\right), F^{*}\left(D_{2}\right)\right)
$$

is nef. Moreover, we have that for any higher model $G: Z \rightarrow Y$,

$$
G^{*}\left(\max \left(F^{*}\left(D_{1}\right), F^{*}\left(D_{2}\right)\right)\right)=\max \left(G^{*} F^{*}\left(D_{1}\right), G^{*} F^{*}\left(D_{2}\right)\right) .
$$

We write $\operatorname{Supp}(D)=\cup_{i} Q_{i}$, where the $Q_{i}$ are prime divisors, and say that

$$
Q_{i} \text { is } \begin{cases}\text { of type } 1, & \text { if } \operatorname{coeff}_{D_{1}} Q_{i}>\operatorname{coeff}_{D_{2}} Q_{i} \\ \text { of type 2, } & \text { if } \operatorname{coeff}_{D_{1}} Q_{i}<\operatorname{coeff}_{D_{2}} Q_{i} \\ \text { of type 0, } & \text { if } \operatorname{coeff}_{D_{1}} Q_{i}=\operatorname{coeff}_{D_{2}} Q_{i} .\end{cases}
$$

Our proof of Theorem 2.2 will be based on the following criterion.

Proposition 2.3. The divisor $\max \left(D_{1}, D_{2}\right)$ is nef if $Q_{i} \cap Q_{j}=\emptyset$ whenever $Q_{i}$ is of type 1 and $Q_{j}$ is of type 2. Moreover, if this condition holds then for any higher model $G: Z \rightarrow X$ then

$$
G^{*}\left(\max \left(D_{1}, D_{2}\right)\right)=\max \left(G^{*}\left(D_{1}\right), G^{*}\left(D_{2}\right)\right)
$$

Proof. We prove first that $\max \left(D_{1}, D_{2}\right)$ is nef. Let $C$ be a curve. We note that at least one of the following holds:

- There is no $Q_{i}$ of type 1 containing $C$,

- There is no $Q_{i}$ of type 2 containing $C$. 
Without loss of generality there is no $Q_{i}$ of type 2 containing $C$. We can write

$$
\max \left(D_{1}, D_{2}\right)=D_{1}+\sum_{i, Q_{i} \text { of type } 2} c_{i} Q_{i},
$$

where the $c_{i}$ s are positive constants. But then

$$
C \cdot \max \left(D_{1}, D_{2}\right)=C \cdot D_{1}+C \cdot\left(\sum_{i, Q_{i} \text { of type } 2} c_{i} Q_{i}\right)
$$

Since $C$ is contained in no $Q_{i}$ of type 2 , the final term is positive and $C \cdot D_{1} \geq 0$ because $D_{1}$ is nef. Hence $C \cdot \max \left(D_{1}, D_{2}\right) \geq 0$ for any curve $C$.

Now, let us prove that given a birational map $G: Z \rightarrow X$ we have that

$$
G^{*}\left(\max \left(D_{1}, D_{2}\right)\right)=\max \left(G^{*}\left(D_{1}\right), G^{*}\left(D_{2}\right)\right) .
$$

We consider a divisor $E \subset Z$. For each $Q_{i}$, let $d_{i} \geq 0$ be the coefficient of $E$ in $G^{*}\left(Q_{i}\right)$. We may assume that $\forall i$ such that $d_{i} \neq 0 Q_{i}$ is of type 1 or 0 . Writing

$$
D_{1}=\sum_{i} a_{i} Q_{i}, D_{2}=\sum_{i} b_{i} Q_{i}
$$

the coefficient of $E$ in $G^{*}\left(\max \left(D_{1}, D_{2}\right)\right)$ is $=\sum_{i} d_{i} \max \left(a_{i}, b_{i}\right)=\sum_{i} d_{i} a_{i}$ since whenever $d_{i} \neq 0$ $a_{i} \geq b_{i}$. But the coefficient of $E$ in $\max \left(G^{*}\left(D_{1}\right), G^{*}\left(D_{2}\right)\right)=\max \left(\sum_{i} a_{i} d_{i}, \sum_{i} b_{i} d_{i}\right)=\sum_{i} a_{i} d_{i}$ and hence the condition holds.

To establish Theorem 3.1 it will therefore be enough to prove the following result.

Theorem 2.4. Let $X, D_{1}, D_{2}$ be as above. There is a projective birational morphism

$$
F: Y \longrightarrow X
$$

from a normal $\mathbb{Q}$-factorial variety $Y$ with the following property. If

$$
F^{-1}(D)=\cup_{i} Q_{i},
$$

then for any pair $i, j$ such that $Q_{i}$ is of type 1 and $Q_{j}$ is of type 2 we have that $Q_{i} \cap Q_{j}=\emptyset$.

(Here "of type 1", for example, is to be understood with respect to the pair of divisors $F^{*}\left(D_{1}\right)$ and $F^{*}\left(D_{2}\right)$.)

We say that $\left(Q_{i}, Q_{j}\right)$ is a bad pair if $Q_{i}$ is of type $1, Q_{j}$ is of type 2 and $Q_{i} \cap Q_{j} \neq \emptyset$. We note that if $Q_{i}$ is of type 1 (resp.2, resp. 0) in $X$ then the proper transform $\bar{Q}_{i}$ is also of type 1 (resp. 2 resp. 0) in $Y$. Our aim will therefore be to create a blow-up $\hat{X}^{\prime}$ of $X$ along $Q_{i} \cap Q_{j}$ for any bad pair $(i, j)$ such that

- $\overline{Q_{i}}$ and $\overline{Q_{j}}$ are separated in $\hat{X}^{\prime}$ and

- the unique exceptional divisor $E \subset \hat{X}^{\prime}$ is of type 0 .

We relegate the proof of Theorem 2.4 to Section 4 .

\section{Construction of Zariski DeCOMPosition FOR B-Divisors}

We proceed with the actual construction of Zariski decompositions, and prove our main result.

Theorem 3.1. Let $X$ be a $\mathbb{Q}$-factorial normal projective variety over an algebraically closed field, $\mathbf{D}$ an effective $\mathbb{Q}$-b-divisor on $X$. Then there exists a unique decomposition

$$
\mathbf{D}=\mathbf{P}_{\mathbf{D}}+\mathbf{N}_{\mathbf{D}} \text {, }
$$

where $\mathbf{P}_{\mathbf{D}}, \mathbf{N}_{\mathbf{D}}$ are effective $\mathbb{Q}$-b-divisors on $X$, such that

(1) $H^{0}\left(X,\left\lfloor k \mathbf{P}_{\mathbf{D}}\right\rfloor\right)=H^{0}(X,\lfloor k \mathbf{D}\rfloor)$

(2) $\mathbf{P}_{\mathbf{D}}$ is a limit of b-nef b-divisors on every proper birational model $Y \rightarrow X$.

(3) For any nef b-divisor $\mathbf{P}^{\prime} \leq \mathbf{D}$ we have that $\mathbf{P}^{\prime} \leq\left(\mathbf{P}_{\mathbf{D}}\right)$. 
Granting Theorem 2.4, we show how to prove Theorem 3.1 and construct a Zariski decomposition in the sense of b-divisors. We start by recalling the definition of a nef b-divisor.

Definition 3.2. Let $\mathbf{P}$ be a b-divisor on $X$. We say that $\mathbf{P}$ is nef if there is a birational model $X^{\prime} \rightarrow X$ such that

- $P \stackrel{\text { def }}{=} \mathbf{P}_{X^{\prime}}$ is nef,

- $\mathbf{P}=\bar{P}$, the Cartier closure of $P$.

We are now going, given a $\mathbb{Q}$-b-divisor $\mathbf{D}$ on $X$, to define the positive part of $\mathbf{D}$.

Definition 3.3. We set

$$
\mathbf{P}_{\mathbf{D}} \stackrel{\text { def }}{=} \max \{\mathbf{P} \mid \mathbf{P} \text { a nef } \mathbb{Q} \text {-b-divisor }, \mathbf{P} \leq \mathbf{D}\} .
$$

After finishing this paper, we learnt that a very similar construction has been used by Boucksom, Favre and Jonsson in their paper [4. More precisely, in the case where $\mathbf{D}$ is a Cartier big divisor, our definition is the same as Boucksom, Favre and Jonsson's definition of a positive intersection product, in the special case where the set of multiplied divisors contains only one element. (See Definition 2.5 and section 3.4 in [4] for more information.)

Then $\mathbf{P}_{\mathbf{D}}$ is a well-defined b-divisor on $X$, and $0 \leq \mathbf{P}_{\mathbf{D}} \leq \mathbf{D}$. In order to prove Theorem 3.1 . we need two preliminary lemmas.

Lemma 3.4. Let $\mathbf{P}_{1}$ and $\mathbf{P}_{2}$ be nef $\mathbb{Q}$-b-divisors. Then $\max \left(\mathbf{P}_{1}, \mathbf{P}_{2}\right)$ is again a nef $\mathbb{Q}$-b-divisor.

Proof. After suitable blow-up, we may assume that $\mathbf{P}_{i}=\overline{P_{i}}$ is the Cartier closure of a nef divisor $P_{i}$ on $X$. Theorem 2.2 says that we may further assume that

$$
P \stackrel{\text { def }}{=} \max \left(P_{1}, P_{2}\right)
$$

is nef and that on any higher model $G: Z \rightarrow X$

$$
\max \left(G^{*} P_{1}, G^{*} P_{2}\right)=G^{*}(P) .
$$

Alternatively, $\max \left(\mathbf{P}_{\mathbf{1}}, \mathbf{P}_{2}\right)=\bar{P}$ and hence $\max \left(\mathbf{P}_{1}, \mathbf{P}_{2}\right)$ is a nef divisor.

Throughout the following, set

$$
\mathbf{M}_{k}(\mathbf{D}) \stackrel{\text { def }}{=} \mathbf{D}-\frac{1}{k} \operatorname{Fix}(k \mathbf{D})
$$

Lemma 3.5. Let $\mathbf{D}$ be a $\mathbb{Q}$-b-divisor on $X$. Then $\mathbf{M}_{k}(\mathbf{D})$ is a nef $\mathbb{Q}$-b-divisor.

Proof. Let $D$ be the trace of $\mathbf{D}$ on $X$. Set $V=H^{0}(X,\lfloor k \mathbf{D}\rfloor) \subset H^{0}(X,\lfloor k D\rfloor)$. By Hironaka's resolution of singularities there is a model $F: Y \rightarrow X$ such that the mobile part of the linear system $V$ on $Y$ is base-point-free, ie. we can write

$$
k \mathbf{D}_{Y}=M_{Y}+F_{Y}
$$

in such a way that $V \subset H^{0}\left(Y, M_{Y}\right)$ and $V$ is base point free as a linear system in $H^{0}\left(Y, M_{Y}\right)$. We note that $\mathbf{M}_{k}(\mathbf{D})_{Y}=\frac{1}{k} M_{Y}$ and that $M_{Y}$ is nef. Since $V \subset H^{0}\left(Y, M_{Y}\right)$ is base-point free we have that

on any higher model $G: Z \rightarrow Y$ and hence

$$
\operatorname{Fix}(k \mathbf{D})_{Z}=k \mathbf{D}_{Z}-G^{*}\left(M_{Y}\right)
$$

$$
\mathbf{M}_{k}(\mathbf{D})=\overline{M_{Y}}
$$

It follows that $\mathbf{M}_{k}(\mathbf{D})$ is a nef b-divisor.

Proof of Theorem 3.1. First we prove that we have $H^{0}\left(X,\left\lfloor k \mathbf{P}_{\mathbf{D}}\right\rfloor\right)=H^{0}(X,\lfloor k \mathbf{D}\rfloor)$ for any $k$. By Lemma 3.5, $\mathbf{M}_{k}(\mathbf{D})$ is a nef $\mathbb{Q}$-b-divisor. It follows by definition that $\mathbf{P}_{\mathbf{D}} \geq \mathbf{M}_{\mathbf{k}}(\mathbf{D})$ and hence that

$$
H^{0}\left(X,\left\lfloor k \mathbf{P}_{\mathbf{D}}\right\rfloor\right)=H^{0}(X,\lfloor k \mathbf{D}\rfloor) .
$$

Condition 3 is satisfied by definition of $\mathbf{P}_{\mathbf{D}}$. It remains to prove condition 2 . Here is what we will prove. 
Claim. For any birational model $X^{\prime} \rightarrow X$, there is a sequence $\mathbf{N}_{n}$ of nef $\mathbb{Q}$ - $b$-divisors such that $\lim _{n \rightarrow \infty}\left(\mathbf{N}_{n}\right)_{X^{\prime}}=\left(\mathbf{P}_{\mathbf{D}}\right)_{X^{\prime}}$

To this end, set $\mathbf{P}_{\mathbf{D} X^{\prime}}=\sum_{i} c_{i} Q_{i}$, the sum being taken over some finite set of irreducible divisors. Let $\epsilon$ be a positive real number. It will be enough to show that there is some nef b-divisor $\mathbf{N}_{\epsilon}$ such that

$$
\left\|\left(\mathbf{P}_{\mathbf{D}}\right)_{X^{\prime}}-(\mathbf{N})_{X^{\prime}}\right\| \leq \epsilon
$$

in the supremum norm. Set $c_{i}-\epsilon=d_{i}$. By definition of $\mathbf{P}_{\mathbf{D}}$ there exists for every $i$ a nef divisor $\mathbf{N}_{i}$ on a model $X_{i}$ such that $\mathbf{N}_{i} \leq \mathbf{D}_{X_{i}}$, and coeff $N_{i}\left(Q_{i}\right) \geq d_{i}$.

By Lemma 3.4

$$
\mathbf{N}_{\epsilon}=\max \left(\bar{N}_{i}\right)
$$

is nef and $\leq \mathbf{D}$. It then follows that $\mathbf{N}_{\epsilon} \leq \mathbf{P}_{\mathbf{D}}$. Since we also have coeff ${ }_{N}\left(Q_{i}\right) \geq c_{i}-\epsilon$ for all $i$, it follows that

$$
\left\|\left(\mathbf{P}_{\mathbf{D}}\right)_{X^{\prime}}-\left(\mathbf{N}_{\epsilon}\right)_{X^{\prime}}\right\| \leq \epsilon
$$

and the Theorem follows.

Since in the case of smooth surfaces there is no need for birational modifications, we get back the Cartier closure of the original Zariski decomposition. Going to higher dimensions, by uniqueness we obtain the following.

Corollary 3.6. Let $D$ be a $\mathbb{Q}$-Cartier divisor on $X$ having a Zariski decomposition $D=P_{D}+N_{D}$ in the sense of Fujita, let $\bar{D}=\mathbf{P}_{\bar{D}}+\mathbf{N}_{\bar{D}}$ be the b-divisorial Zariski decomposition of the Cartier closure $\bar{D}$. Then

$$
\mathbf{P}_{\bar{D}}=\overline{P_{D}}, \quad \text { and } \mathbf{N}_{\bar{D}}=\overline{N_{D}} .
$$

In the special case where $\mathbf{D}$ is big, we can do better. Recall that a b-divisor is called big if it is the Cartier closure of a big divisor on some model.

Proposition 3.7. If $\mathbf{D}$ is big, then $\mathbf{P}_{\mathbf{D}}=\lim _{m \rightarrow \infty} \mathbf{M}_{m}(\mathbf{D})$.

Proof. Let $\mathbf{N}$ be nef and $\leq \mathbf{D}$. Choose a $k$ such that $\mathbf{M}_{k}(\mathbf{D})$ is big as well as nef. By Lemma 3.4. $\mathbf{M}_{k}^{\prime}(\mathbf{D})=\max \left(\mathbf{N}, \mathbf{M}_{k}(\mathbf{D})\right)$ is big and nef. Blowing-up, we may assume that $\mathbf{M}_{k}^{\prime}(\mathbf{D})$ is the Cartier closure of its trace on $X, \overline{M_{k}^{\prime}(D)}$. By Wilson's result [21]

But now

$$
\lim _{m \rightarrow \infty}\left(\mathbf{M}_{k}^{\prime}(\mathbf{D})-\frac{1}{m} \operatorname{Fix}\left(m \mathbf{M}_{k}^{\prime}(\mathbf{D})\right)\right)=\mathbf{M}_{k}^{\prime}(\mathbf{D}) \text {. }
$$

$$
\mathbf{N} \leq \mathbf{M}_{k}^{\prime}(\mathbf{D})=\lim _{m \rightarrow \infty}\left(\mathbf{M}_{k}^{\prime}(\mathbf{D})-\frac{1}{m} \operatorname{Fix}\left(m \mathbf{M}_{k}^{\prime}(\mathbf{D})\right)\right) \leq \lim _{m \rightarrow \infty} \mathbf{M}_{m}(\mathbf{D}) \leq \mathbf{P}_{\mathbf{D}}
$$

where the last inequality is valid because $\mathbf{P}_{\mathbf{D}}$ carries all the sections of $\mathbf{D}$. Hence

$$
\mathbf{N} \leq \lim _{m \rightarrow \infty} \mathbf{M}_{m}(\mathbf{D}) \leq \mathbf{P}_{\mathbf{D}}
$$

for any nef sub-divisor $\mathbf{N}$ of $\mathbf{D}$. Since $\mathbf{P}_{\mathbf{D}}$ is simply the maximum of all such $\mathbf{N}$ 's, it follows that

$$
P_{D}=\lim _{m \rightarrow \infty} \mathbf{M}_{m}(\mathbf{D}) \text {. }
$$

In this case, in particular, $\mathbf{P}_{\mathbf{D}}$ is a limit in the strong sense of nef $b$-divisors.

Although the positive part of a b-divisorial Zariski decomposition is not nef, it shares many of the important properties of nef divisors, vanishing being one of the most important.

Corollary 3.8 (Vanishing Theorem). Let $\mathbf{D}$ be a big b-divisor on a smooth variety $X$. then

$$
H^{i}\left(X, \mathcal{O}_{X}\left(K_{X}\right) \otimes \mathcal{O}_{X}\left(\mathbf{P}_{\mathbf{D}}\right)\right)=0
$$

for all $i \geq 1$.

Proof. In this case, we have $\mathcal{O}_{X}\left(-\mathbf{N}_{\mathbf{D}}\right)=\mathcal{J}(\|D\|)$, the multiplier ideal of $D$, so this is just another restatement of Nadel vanishing. 
4. The Blow-up Separating $Q_{i}$ And $Q_{j}$.

We move on to proving Theorem 2.4, the technical core of the paper. It will be useful to change conventions slightly: from now on, the set of divisors

$$
S\left(X, D_{1}, D_{2}\right)=\left\{Q_{1} \ldots Q_{r}\right\}
$$

will consist of all divisors in the support of $D$ which are of type 1 or type 2 . In other words, we remove from this set all the divisors of type 0 .

We make the following assumptions.

Assumption 4.1 .

(1) $X$ is a $\mathbb{Q}$-factorial normal variety.

(2) For any $m$-tuple $\left(k_{1}, k_{2}, \ldots, k_{m}\right)$ the intersection $Q_{k_{1}} \cap \ldots \cap Q_{k_{m}}$ is of pure codimension $m$.

(3) For any pair of distinct $(i, j)$ and for a sufficiently general point $x \in Q_{i} \cap Q_{j}, x$ is a smooth point of $Q_{i}, Q_{j}$ and $X$ and $Q_{i}$ and $Q_{j}$ intersect transversally at $x$.

(4) For any pair of distinct $(i, j), Q_{i} \cap Q_{j}$ is irreducible.

Proposition 4.2. Under assumptions 4.1, for any bad pair $\left(Q_{i}, Q_{j}\right)$, there is a proper birational morphism $\mu: \tilde{X} \rightarrow X$ with a unique exceptional divisor $E$ such that:

(1) $E$ is of type 0 (relative to $\mu^{*}\left(D_{1}\right)$ and $\mu^{*}\left(D_{2}\right)$ ),

(2) $\bar{Q}_{i}$ and $\bar{Q}_{j}$ do not meet in $\tilde{X}$,

(3) the conditions of 4.1 are valid for $\tilde{X}$ and $S\left(\tilde{X}, \mu^{*}\left(D_{1}\right), \mu^{*}\left(D_{2}\right)\right)=\left\{\bar{Q}_{1} \ldots \bar{Q}_{r}\right\}$.

Such a blow-up will be called a separating blow-up for $\left(Q_{i}, Q_{j}\right)$. We start by showing that Theorem 2.4 follows immediately from Proposition 4.2 .

Proof of Theorem 2.4. After a possible initial blow-up, we may assume that conditions of 4.1 are satisfied. Let $\mu: X \rightarrow X$ be a morphism whose existence is guaranteed by Proposition 4.2 Then the set of bad pairs for $\left(\tilde{X}, \mu^{*}\left(D_{1}\right), \mu^{*}\left(D_{2}\right)\right)$ is a subset of

$$
\left\{\left(\bar{Q}_{k_{1}}, \bar{Q}_{k_{2}}\right) \mid\left(Q_{k_{1}}, Q_{k_{2}}\right) \text { is a bad pair for }\left(X, D_{1}, D_{2}\right),\left(k_{1}, k_{2}\right) \neq(i, j)\right\} \text {. }
$$

In particular, the number of bad pairs strictly decreases under a separating blow-up. Iterating this procedure, we produce a proper birational map $F: Y \rightarrow X$ such that $\left(Y, F^{*}\left(D_{1}\right), F^{*}\left(D_{2}\right)\right)$ has no bad pairs and $1-4$ holds for $Y$. But then $F: Y \rightarrow X$ is exactly the map we seek in Theorem 2.4

To be able to proceed with the proof of Proposition 4.2. we start by defining the type of birational modification we need. Let $a, b$ be positive coprime integers. We now define an " $(a, b)$ blow-up along the pair $\left(Q_{i}, Q_{j}\right) "$.

Choose an integer $m$ such that $m Q_{i}$ and $m Q_{j}$ are both Cartier. Denote

$$
\pi: \hat{X} \stackrel{\text { def }}{=} \mathrm{Bl}_{I_{i}^{a}+I_{j}^{b}} X \longrightarrow X
$$

where $I_{i}$ and $I_{j}$ denote the ideal sheaves $\mathcal{O}_{X}\left(-m Q_{i}\right)$, and $\mathcal{O}_{X}\left(-m Q_{j}\right)$, respectively. As a consequence of [10, Proposition 7.16.] $\hat{X}$ is a variety, in particular it is an integral scheme.

Remark 4.3. The blow-up constructed above can be given explicitly in local terms as follows. Choose open affines $U_{k} \subset X$ such that $\left(m Q_{i}\right) \cap U_{k}$ is defined by a single function $f_{k}$ and $\left(m Q_{j}\right) \cap U_{k}$ is defined by a function $g_{k}$. We define $\hat{U}_{k}$ of $U_{k}$

$$
\hat{U}_{k}=\left\{(x,[U: V]) \in U_{k} \times \mathbb{P}^{1} \mid U g_{k}^{b}=V f_{k}^{a}\right\},
$$

where we understand $\hat{U}_{k}$ to be the subscheme of $U_{k} \times \mathbb{P}^{1}$ defined by this equation. These open sets can be glued together to give the global blow-up scheme $\hat{X}$. 
The variety $\hat{X}$ is not immediately useful, since it is not normal. Consider the normalisation $\hat{X}^{\prime}$ of $\hat{X}$ in the function field $K(\hat{X})$. We denote the normalisation map by $n: \hat{X}^{\prime} \rightarrow \hat{X}$, and the composition $\pi \circ n$ by $\pi^{\prime}$. Note that $\pi$ is proper (since projective) and birational and that $n$ is proper and birational. Therefore, $\pi^{\prime}$ is proper and birational.

Throughout what follows the normalisation of an open set $A \subset \hat{X}$ will be denoted by $A^{\prime} \subset \hat{X}^{\prime}$.

Proposition 4.4. The map $\pi^{\prime}: \hat{X}^{\prime} \rightarrow X$ has a unique exceptional divisor.

In the course of this proof, we will also find explicit equations for a certain open set $\hat{X}^{\prime}$, which will be useful later on.

Proof of Proposition 4.4. The exceptional locus of $\pi: \hat{X} \rightarrow X$ is a $\mathbb{P}^{1}$ bundle over the irreducible set $Q_{i} \cap Q_{j}$, so it only contains one exceptional divisor, call it $E_{1}$.

Any exceptional divisor in $\hat{X}^{\prime}$ maps to $E_{1}$ under $n$. It will therefore be enough to show that $n^{-1}\left(E_{1}\right)$ contains only one divisor. Moreover, since the normalisation map is finite-to-one, it will be enough to find some open set, $\hat{U} \subset \hat{X}$, meeting $E_{1}$, such that $n^{-1}\left(E_{1} \cap U\right)$ contains a unique divisor in $n^{-1}(\hat{U})$.

We choose an open affine set $W \subset X$ such that $W$ is smooth and $Q_{i} \cap W$ and $Q_{j} \cap W$ are smooth and meet transversally. Such $W$ exists, since $\hat{X}^{\prime}$ is normal. We assume further that there are regular functions $f$ and $g$ on $W$ such that $Q_{i}=\operatorname{Zero}(f)$ and $Q_{j}=\operatorname{Zero}(g)$.

One possible projective embedding of $\hat{W}=\pi^{-1}(W)$ is

$$
\hat{W}=\left\{(x,[U: V]) \in W \times \mathbb{P}^{1} \mid U g^{m b}=V f^{m a}\right\} .
$$

We consider the open affine set $\hat{U} \subseteq \hat{W}$ given by

$$
\hat{U}=\{(x,[U: V]) \in \hat{W} \mid U, V \neq 0\},
$$

which we can also write as

$$
\hat{U}=\left\{(x, u) \in W \times\left(\mathbb{A}^{1} \backslash\{0\}\right) \mid u g^{m b}=f^{m a}\right\} .
$$

We note first of all that the rational function on $\hat{U}$ given by $s=\frac{f^{a}}{g^{b}}$ satisfies $s^{m}=u$ and is hence a regular function on $\hat{U}^{\prime}$, the normalisation of $\hat{U}$.

Let us now consider the integral affine scheme

$$
\hat{U}_{1}=\left\{(x, s) \in W \times\left(\mathbb{A}^{1} \backslash\{0\}\right) \mid s g^{b}=f^{a}\right\} .
$$

There is a natural surjective map $\theta: \hat{U}_{1} \rightarrow \hat{U}$ given by $\theta(x, s)=\left(x, s^{m}\right)$. This is an isomorphism over the open set $\hat{U} \backslash E_{1} \cap \hat{U}$, so there is an inclusion $A\left(\hat{U}_{1}\right) \subset K(\hat{U})$ : since all elements of $A\left(\hat{U}_{1}\right)$ are integral over $A(\hat{U})$ it follows that there are maps

$$
\hat{U}^{\prime} \stackrel{\mu}{\rightarrow} \hat{U}_{1} \stackrel{\theta}{\rightarrow} \hat{U}
$$

such that $\theta \circ \mu=n$.

In other words, $\hat{U}^{\prime}$ is also the normalisation of $\hat{U}_{1}$, which however is still not normal: we need to add some extra regular functions.

Choose numbers $(c, d)$ such that $b d-a c=1$. Consider the element

$$
t \stackrel{\text { def }}{=} \frac{f^{d}}{g^{c}} \in K\left(\hat{U}_{1}\right) \text {. }
$$

We note that

$$
t^{a}=\frac{f^{a d}}{g^{a c}}=s^{d} g .
$$

Similarly $t^{b}=s^{c} f$, and in particular $t \in A\left(\hat{U}^{\prime}\right)$. We now consider the scheme defined as follows.

$$
\hat{U}_{2}=\left\{(x, s, t) \in \hat{U} \times\left(\mathbb{A}^{1} \backslash\{0\}\right) \times \mathbb{A}^{1} \mid t^{a}=s^{d} g, t^{b}=s^{c} f\right\} .
$$

In $\hat{U}_{2}$ we have that

$$
s^{b d} g^{b}=t^{a b}=s^{a c} f^{a}=s^{b d-1} f^{a}
$$


and that

$$
s^{c d} g^{c} t=t^{a c+1}=t^{b d}=s^{c d} f^{d} .
$$

so it follows that $s g^{b}=f^{a}$ and $t g^{c}=f^{d}$ in $A\left(\hat{U}_{2}\right)$. In particular, there is a natural map

$$
\nu: \hat{U}_{2} \rightarrow \hat{U}_{1}
$$

given by $\nu(x, s, t)=(x, s)$. We note that $\nu$ is surjective and set-theoretically one-to-one. Indeed, for any $(x, s) \in \hat{U}_{1}$,

$$
\nu^{-1}(x, s)=\left\{(x, s, t) \mid t^{a}=s^{d} g, t^{b}=s^{c} f\right\} .
$$

and it easy to see that for fixed $x, s$ such that $s g^{b}=F^{a}$ these equations have exactly one solution in $t$. We note further that as sets

$$
E_{2} \stackrel{\text { def }}{=}(\theta \circ \nu)^{-1}\left(E_{1}\right)=\left(Q_{i} \cap Q_{j} \cap W\right) \times\left(\mathbb{A}^{1} \backslash\{0\}\right) \times\{0\},
$$

and hence this set contains only one divisor. We aim now to show that $\hat{U}_{2}$ is in fact the normalisation of $\hat{U}$.

Lemma 4.5. $\hat{U}_{2}$ is smooth and everywhere of dimension n. Moreover, at all points of $E_{2} \cap \hat{U}_{2} t$ is a local equation for the divisor $E_{2}=(\theta \circ \nu)^{-1}\left(E_{1}\right)$.

Proof. Let $(x, s, t)$ be a point of $\hat{U}_{2}$ with $x \in W$. We consider $W$ as a subset of an affine space $\mathbb{A}^{M}$. Let $x_{1} \ldots x_{m}$ be the local coordinates on $\mathbb{A}^{M}$, and let $h_{1}, \ldots h_{k}$ be local equations for $W$ at $x$. The assumption that $x$ should be a smooth point of $W$ at which $Q_{i}$ and $Q_{j}$ are smooth and meet transversally means that the vectors

$$
\left\{\left(\begin{array}{c}
\frac{\partial h_{1}}{\partial x_{1}} \\
\vdots \\
\frac{\partial h_{1}}{\partial x_{m}}
\end{array}\right), \ldots,\left(\begin{array}{c}
\frac{\partial h_{k}}{\partial x_{1}} \\
\vdots \\
\frac{\partial h_{k}}{\partial x_{m}}
\end{array}\right),\left(\begin{array}{c}
\frac{\partial f}{\partial x_{1}} \\
\vdots \\
\frac{\partial f}{\partial x_{m}}
\end{array}\right),\left(\begin{array}{c}
\frac{\partial g}{\partial x_{1}} \\
\vdots \\
\frac{\partial g}{\partial x_{m}}
\end{array}\right)\right\}
$$

are linearly independent. (The implicit evaluations at $x$ have been omitted for legibility's sake.) $\hat{U}_{2}$ is a subset of $\mathbb{A}^{M} \times\left(\mathbb{A}^{1} \backslash\{0\}\right) \times \mathbb{A}^{1}$ given by the set of equations

$$
h_{1} \ldots h_{k}, t^{a}-s^{d} g, t^{b}-s^{c} f
$$

It follows from the Jacobian criterion that $\hat{U}_{2}$ is smooth and of dimension $n$ everywhere. Moreover,

$$
t(y)=0 \Leftrightarrow f(y)=g(y)=0
$$

and hence $E_{2}$ is set-theoretically given by the equation $t=0$. The Jacobian criterion also shows that $d t \neq 0$ in $\Omega_{\hat{U}^{2}}^{1}$ at any point $x \in \hat{U}_{2}$ and it follows that $t$ is a local equation for $E_{2}$.

Let us show that $\hat{U}_{2}$ is integral. It is enough to show that it is not a disjoint union of disconnected components. But this follows from the fact that $\nu$ is one-to-one and that every component of $\hat{U}_{2}$ has dimension $n=\operatorname{dim}\left(\hat{U}_{1}\right)$.

We now show that the normalisation map factors through $\nu: \hat{U}_{2} \rightarrow \hat{U}_{1}$. Over the points where $g \neq 0$ we can write

$$
t=\frac{t^{b d}}{t^{a c}}=\frac{s^{c d} f^{d}}{s^{d c} g^{c}} \in A\left(\hat{U}_{1}\right),
$$

so there is an open set over which $\nu$ is an isomorphism. Hence there is an inclusion $A\left(\hat{U}_{2}\right) \subset K\left(\hat{U}_{1}\right)$. Moreover, $\hat{U}_{2}$ is integral over $\hat{U}_{1}$. It follows that there is a factorisation

$$
\hat{U}^{\prime} \stackrel{\phi}{\rightarrow} \hat{U}_{2} \stackrel{\nu}{\rightarrow} \hat{U}_{1}
$$

such that $\nu \circ \phi=\mu$, and such that $\hat{U}^{\prime}$ is the normalisation of $\hat{U}_{2}$. But since $\hat{U}_{2}$ is smooth and hence normal, $\phi$ is an isomorphism.

It follows that $\hat{U}^{\prime}$ has a unique exceptional divisor over $W, \phi^{-1}\left(E_{2}\right)$, and hence that $\hat{X}^{\prime}$ indeed contains a unique exceptional divisor, $\overline{\phi^{-1}\left(E_{2}\right)}$, which we denote by $E$.

We will now show that the $(a, b)$ blow-up has good properties. 
Lemma 4.6. Suppose that $Q_{i}$ is of type 1 and $Q_{j}$ is of type 2. For a suitable choice of $(a, b)$, the coefficient of $E$ in $\pi^{\prime *} D_{1}$ is the same as its coefficient in $\pi^{\prime *} D_{2}$.

Proof. In the above notation, $t$ is a local equation for $E$ at a generic point of $E$. Let $f$ be a local equation for $Q_{i}$. We have seen above that at a generic point of $E, t^{b}=s^{c} f$, so $E$ appears with coefficient $b$ in $\pi^{\prime *}\left(Q_{i}\right)$. Likewise, $E$ appears with coefficient $a$ in $\pi^{\prime *}\left(Q_{j}\right)$.

Now, since $Q_{i}$ is of type 1 and $Q_{j}$ is of type 2 we can write

$$
D_{1}=M+c_{1} Q_{i}+F_{1}, D_{2}=M+c_{2} Q_{j}+F_{2}
$$

where

- $M$ is the minimum of $D_{1}$ and $D_{2}$,

- the $c_{i}$ 's are positive rationals,

- $F_{1}$ and $F_{2}$ are divisors whose support does not contain $Q_{i} \cap Q_{j}$.

In particular, $F_{1}$ and $F_{2}$ do not contribute to the coefficient of $E$ in $\pi^{\prime *}\left(D_{i}\right)$. It is therefore enough to require $c_{1} b=c_{2} a$. In other words, by picking $(a, b)$ to be the unique pair of coprime positive integers such that $a / b=c_{1} / c_{2}$, we can arrange the required coefficients to be equal.

We now need the following proposition.

Proposition 4.7. The divisors $\bar{Q}_{i}$ and $\bar{Q}_{j}$ do not meet in $\hat{X}^{\prime}$.

Proof. It will be enough to show that $\bar{Q}_{i}$ and $\bar{Q}_{j}$ do not meet in $\hat{X}$. But for any $k \bar{Q}_{i} \cap \hat{U}_{k}$ is contained in the set given by $U=0$ and $\bar{Q}_{j} \cap \hat{U}_{k}$ is contained in the set given by $V=0$, which are disjoint.

Henceforth, we will call any $(a, b)$ blow-up along $\left(Q_{i}, Q_{j}\right)$ such that the coefficient of $E$ is the same in $\pi^{*}\left(D_{1}\right)$ as in $\pi^{*}\left(D_{2}\right)$ a separating blow-up for $(i, j)$. In particular, if $\left(\hat{X}^{\prime}, \pi^{\prime}\right)$ is a separating blow-up for $\left(Q_{i}, Q_{j}\right)$ then $\pi^{\prime}$ has a unique exceptional divisor of type 0 and that $\bar{Q}_{i}$ and $\bar{Q}_{j}$ do not meet in $\hat{X}^{\prime}$.

Proof of Proposition 4.2. Choose $(a, b)$ such that the conditions of Lemma 4.6 are fulfilled, let $\tilde{X}=\hat{X}^{\prime}$ and $\mu=\pi^{\prime}$ for this pair $(a, b)$. According to Proposition 4.7 the morphism $\mu$ is a separating blow-up for the pair $\left(Q_{i}, Q_{j}\right)$ provided the assumptions 4.1 are satisfied.

We start with proving that $\hat{X}^{\prime}$ is $\mathbb{Q}$-factorial. We have that

$$
\mathbb{Q W e i l}\left(\hat{X}^{\prime}\right)=\pi^{*}(\mathbb{Q W e i l}(X)) \oplus\langle E\rangle \text {. }
$$

We are done if we can show that $E$ is a $\mathbb{Q}$-Cartier divisor. It will be enough to produce a Cartier divisor $L$ on $\hat{X}$ such that (set-theoretically) $\operatorname{Supp}(L)=E_{1}$. Indeed, the pull-back $n^{*}(L)$ is then a Cartier divisor on $\hat{X}^{\prime}$ whose support is contained in $n^{-1}\left(E_{1}\right)$. But this set contains only one prime divisor, $E$, so the Weil divisor associated to $\pi^{\prime *}\left(E_{1}\right)$ is necessarily a multiple of $E$.

We now construct $L$ as follows. Consider the covering of $\hat{X}$ by the sets $\hat{U}_{k}^{1}$ and $\hat{U}_{k}^{2}$ given by

$$
\begin{gathered}
\hat{U}_{k}^{1}=\left\{(x,[U: 1]) \in \hat{U}_{k}\right\} \\
\hat{U}_{k}^{2}=\left\{(x,[1: V]) \in \hat{U}_{k}\right\} .
\end{gathered}
$$

We choose the Cartier divisor given by $g_{k}^{b}$ on $\hat{U}_{k}^{1}$ and $f_{k}^{a}$ on $\hat{U}_{k}^{2}$. It is immediate that the support of this Cartier divisor is $E_{1}$, hence $\tilde{X}=\hat{X}^{\prime}$ is $\mathbb{Q}$-factorial.

Condition (1) of 4.1 is therefore inherited. Conditions (2)-(4) will quickly follow from the following lemma.

Lemma 4.8. Consider divisors $\bar{Q}_{k_{1}} \ldots, \bar{Q}_{k_{m}}$ in $\hat{X}^{\prime}$. The intersection

$$
\bar{Q}_{k_{1}} \cap \ldots \cap \bar{Q}_{k_{m}} \cap E
$$

is of codimension $\geq m+1$.

Proof. Since $\bar{Q}_{i} \cap \bar{Q}_{j}=\emptyset$ we can assume that either 
(1) $i, j \neq\left\{k_{1}, \ldots, k_{m}\right\}$,

(2) $i \in\left\{k_{1}, \ldots, k_{m}\right\}, j \notin\left\{k_{1} \ldots, k_{m}\right\}$

We consider first the

Case 1. We have that

$$
\bar{Q}_{k_{1}} \cap \ldots \cap \bar{Q}_{k_{m}} \cap E \subset \pi^{\prime-1}\left(Q_{k_{1}} \cap \ldots \cap Q_{k_{m}} \cap Q_{i} \cap Q_{j}\right) .
$$

But $Q_{k_{1}} \cap \ldots \cap Q_{j}$ has codimension $(m+2)$, so $\pi^{\prime-1}\left(Q_{k_{1}} \cap \ldots \cap Q_{k_{m}} \cap Q_{i} \cap Q_{j}\right)$ has codimension $\geq m+1$

Case 2. We assume without loss of generality that $i=k_{1}$. We then have that

$$
\left(\bar{Q}_{k_{1}} \cap \ldots \cap \bar{Q}_{k_{m}} \cap E\right) \subset\left(\overline{Q_{i}} \cap E\right) \cap \pi^{\prime-1}\left(Q_{k_{2}} \cap \ldots \cap Q_{k_{m}}\right)
$$

and

$$
\left(\overline{Q_{i}} \cap E\right) \cap \pi^{\prime-1}\left(Q_{k_{2}} \cap \ldots \cap Q_{k_{m}}\right) \subset\left(E \cap \bar{Q}_{i}\right) \cap \pi^{\prime-1}\left(Q_{k_{2}} \cap \ldots \cap Q_{k_{m}} \cap Q_{i} \cap Q_{j}\right) .
$$

But the map

$$
\pi^{\prime}: E \cap \bar{Q}_{i} \rightarrow Q_{i} \cap Q_{j}
$$

is finite-to-one, so the codimension of

$$
\left(E \cap \bar{Q}_{i}\right) \cap \pi^{\prime-1}\left(Q_{k_{2}} \cap \ldots \cap Q_{k_{m}} \cap Q_{i} \cap Q_{j}\right)
$$

is at least $m+1$ and

$$
\operatorname{codim}\left(Q_{k_{2}} \cap \ldots \cap Q_{k_{m}} \cap Q_{i} \cap Q_{j}\right) \geq m+1 .
$$

But now, every irreducible component of $\bar{Q}_{k_{1}} \cap \ldots \cap \bar{Q}_{k_{m}}$ is of codimension at most $m$, since it is an intersection of $m$ divisors in a $\mathbb{Q}$-factorial normal variety. It follows that

$$
\left(\bar{Q}_{k_{1}} \cap \ldots \cap \bar{Q}_{k_{m}}\right) \cap\left(\pi^{-1}\left(X \backslash\left(Q_{i} \cap Q_{j}\right)\right)\right) \cong Q_{k_{1}} \cap \ldots \cap Q_{k_{m}} \cap\left(X \backslash Q_{i} \cap Q_{j}\right)
$$

is a dense open subset of $\bar{Q}_{k_{1}} \cap \ldots \cap \bar{Q}_{k_{m}}$. Hence (2), (3) and (4) hold for $\hat{X}^{\prime}$. This completes the proof of Proposition 4.2

\section{REFERENCES}

[1] Bauer, T. A simple proof for the existence of Zariski decomposition on surfaces. Journal of Algebraic Geometry, S 1056-3911(08)00509-2, to appear.

[2] Benveniste, X. Sur la décomposition de Zariski en dimension 3. C. R. Acad. Sci. Paris Sér. I Math. 295 (1982), no. 2, 107-110.

[3] Benveniste, X. Sur l'anneau canonique de certaines variétés de dimension 3. Invent. Math. 73 (1983), no. 1, $157-164$.

[4] Boucksom, S., Favre, C., Jonsson, M. Differentiability of divisors and a problem of Teissier. math.AG/0608260

[5] Flips for 3-folds and 4-folds. Edited by Alessio Corti. Oxford Lecture Series in Mathematics and its Applications, 35. Oxford University Press, Oxford, 2007.

[6] Corti, A., Kaloghiros A-S., Ĺazic, V. Introduction to the minimal model program and the existence of flips. Preprint

[7] Cutkosky, S. Zariski decomposition of divisors on algebraic varieties. Duke Mathematical Journal 53 (1986) no. 1., 149-156.

[8] Demailly, J-P.; Peternell, T.; Schneider, M. Pseudo-effective line bundles on compact Kähler manifolds. Internat. J. Math. 12 (2001), no. 6, 689-741.

[9] Fujita, T. Canonical rings of algebraic varieties. Classification of algebraic and analytic manifolds (Katata, 1982), 65-70, Progr. Math., 39, Birkhäuser Boston, Boston, MA, 1983.

[10] Hartshorne, R. Algebraic Geometry. Graduate Texts in Mathematics, No. 52. Springer Verlag, New YorkHeidelberg, 1977.

[11] Kawamata, Y. The Zariski decomposition of log-canonical divisors. Algebraic geometry, Bowdoin, 1985 (Brunswick, Maine, 1985), 425-433, Proc. Sympos. Pure Math., 46, Part 1, Amer. Math. Soc., Providence, RI, 1987

[12] Kollár, J.; Mori, S. Birational geometry of algebraic varieties. With the collaboration of C. H. Clemens and A. Corti. Cambridge Tracts in Mathematics, 134. Cambridge University Press, Cambridge, 1998.

[13] Flips and abundance for algebraic threefolds. Papers from the Second Summer Seminar on Algebraic Geometry held at the University of Utah, Salt Lake City, Utah, August 1991. Astérisque No. 211 (1992). Société Mathématique de France, Paris, 1992. pp. 1-258. 
[14] Moriwaki, A. Semiampleness of the numerically effective part of Zariski decomposition. J. Math. Kyoto Univ. 26 (1986), no. 3, 465-481.

[15] Lazarsfeld, R. Positivity in Algebraic Geometry I.-II. Ergebnisse der Mathematik und ihrer Grenzgebiete, Vols. 48-49., Springer Verlag, Berlin, 2004.

[16] Nakayama, N. Zariski-decomposition and abundance. MSJ Memoirs, 14. Mathematical Society of Japan, Tokyo, 2004.

[17] Prokhorov, Yu. G. On the Zariski decomposition problem. Tr. Mat. Inst. Steklova 240 (2003), 43-72; translation in Proc. Steklov Inst. Math. 2003, no. 1 240, 37-65

[18] Shokurov, V. V. Prelimiting flips. Tr. Mat. Inst. Steklova 240 (2003), 82-219; translation in Proc. Steklov Inst. Math. 2003, no. 1 240, 75-213

[19] Tsuji, H. Analytic Zariski decomposition. International Symposium "Holomorphic Mappings, Diophantine Geometry and Related Topics" (Kyoto, 1992).

[20] Tsuji, H. Canonical singular hermitian metrics on relative canonical bundles. arXiv:0704.0566

[21] P. M. H. Wilson: On the canonical ring of algebraic varieties, Compositio Math. 43 (1981), no. 3, 365-385.

[22] Zariski, O. The theorem of Riemann-Roch for high multiples of an effective divisor on an algebraic surface. Annals of Mathematics (2) 56 (1962), 560-615.

Universität Duisburg-Essen, Campus Essen, FB 6 Mathematik, D-45117 Essen, Germany

Budapest University of Technology and Economics, Budapest P.O. Box 91, H-1521 Hungary

E-mail address: alex.kueronya@uni-due.de

Université Joseph Fourier, UfR de Mathématiques, 100 rue des Maths, BP 74, 38402 St Martin D'Héres, France

E-mail address: Catriona.Maclean@ujf-grenoble.fr 